Journal of Zhejiang University-SCIENCE B (Biomedicine \& Biotechnology)

ISSN 1673-1581 (Print); ISSN 1862-1783 (Online)

www.zju.edu.cn/jzus; www.springerlink.com

E-mail: jzus@zju.edu.cn

\title{
Erratum:
}

\section{Erratum to: Identification of a novel mutation in a Chinese family with Nance-Horan syndrome by whole exome sequencing}

\author{
Nan $\mathrm{HONG}^{\S 1}$, Yan-hua $\mathrm{CHEN}^{\S 2}$, Chen XIE ${ }^{1}$, Bai-sheng $\mathrm{XU}^{1}$, Hui HUANG ${ }^{2}, \mathrm{Xin}_{\mathrm{LI}^{2}}$, Yue-qing YANG ${ }^{2}$, \\ Ying-ping $\mathrm{HUANG}^{2}$, Jian-lian $\mathrm{DENG}^{2}$, Ming $\mathrm{QI}^{\dagger 2,3,4}$, Yang-shun $\mathrm{GU}^{\dagger+1}$ \\ ( ${ }^{I}$ Department of Ophthalmology, the First Affiliated Hospital, School of Medicine, Zhejiang University, Hangzhou 310003, China) \\ ( ${ }^{2}$ Beijing Genomics Institute (BGI)-Shenzhen, Shenzhen 518083, China) \\ $\left({ }^{3}\right.$ School of Basic Medical Sciences, Zhejiang University, Hangzhou 310058, China) \\ $\left({ }^{4}\right.$ Functional Genomics Center, Department of Pathology \& Laboratory Medicine, \\ University of Rochester Medical Center, West Henrietta, NY 14586, USA) \\ †E-mail: mingqi@zju.edu.cn; guyangshun_1@hotmail.com
}

doi: $10.1631 /$ jzus.B13e0321

Erratum to: J Zhejiang Univ-Sci B (Biomed \& Biotechnol) 2014 15(8):727-734

doi:10.1631/jzus.B1300321

The original version of this article unfortunately contained a mistake. In the authors "Nan $\mathrm{HONG}^{\text {§1 }}$, Yan-hua $\mathrm{CHEN}^{\S 1}$, Chen XIE ${ }^{1}$, Bai-sheng XU ${ }^{1}$, Hui $\mathrm{HUANG}^{2}$, Xin $\mathrm{LI}^{2}$, Yue-qing YANG ${ }^{2}$, Ying-ping $\mathrm{HUANG}^{2}$, Jian-lian DENG ${ }^{2}$, Ming QI ${ }^{\dagger+2,3,4}$, Yangshun $\mathrm{GU}^{\dagger \neq 1 ",}$, the superscript sequence number "1", which marked the affiliation of "Yan-hua CHEN", was incorrect. The correct version should be "Nan $\mathrm{HONG}^{\S 1}$, Yan-hua $\mathrm{CHEN}^{\S 2}$, Chen XIE ${ }^{1}$, Bai-sheng $\mathrm{XU}^{1}$, Hui $\mathrm{HUANG}^{2}$, Xin $\mathrm{LI}^{2}$, Yue-qing $\mathrm{YANG}^{2}$, Ying-ping $\mathrm{HUANG}^{2}$, Jian-lian $\mathrm{DENG}^{2}$, Ming $\mathrm{QI}^{\dagger \ddagger 2,3,4}$, Yang-shun $\mathrm{GU}^{\dagger \Uparrow 1}$.

The online version of the original article can be found at http://dx.doi.org/10.1631/jzus.B1300321

${ }^{\ddagger}$ Corresponding authors

$\S$ The two authors contributed equally to this work

(C) Zhejiang University and Springer-Verlag Berlin Heidelberg 2014 